\title{
Kongreßkalender 1998 und weitere Vorschau
}

Für dic Aufnahme neuer Veranstaltungen in den Kongreßkalender senden Sie bitte die entsprechenden Informationen an:

Dr. Francesco Dati
Dade Behring
Scientific Affairs Chemistry
Postfach / P.O. Box 1149
D-35001 Marburg/Germany
Tel.: (49)-6421-39-2958
Fax: (49)-6421-39-3126

Für eine ausführliche Liste wissenschaftlicher Veranstaltungen siehe Laboratoriums Medizin, Heft 3/1998.

May 6 - 9, 1998

Berlin, Germany

May 8 - 10, 1998

Munich, Germany

May $11-15,1998$

Glasgow, UK

May 21 - 23, 1998

Leipzig, Germany

May $21-25,1998$

Erfurt, Germany

June 3 - 5, 1998

Berlin, Germany

June 6 - 10, 1998

Turku, Finland

June 8 - 12, 1998

Berlin, Germany

June 12 - 13, $1998^{\circ}$

Hamburg, Germany

June 14 - 17, 1998

Lugano, Switzerland

\section{2nd Congress of Molecular Medicine}

Secr.: Springer Congress GmbH, Heidelberger Platz 3, D-14197 Berlin, Germany,

Tel.: (49) 3082787 431; Fax: (49) 3082787 465. E-Mail: gansebohm@springerkongress.de, Internct: http://www.cmm.springer.de

\section{5th International Symposium on Metal Ions in Biology \& Medicine}

Secr: Ms. U. Schrödel, GSF Forschungszentrum-Congress Service, Ingolstädter Landstraße 1, D-85764 Oberschleißheim. Germany, Tel.: (49) 89 3187 3030; Fax: (49) 8931873362 ,

E-Mail: schroedel@gsf.de, Internet: http://www.hmi.de/bereiche/N/NG/Events.html

\section{Focus 98 - National Scientific Meeting of the Association of Clinical Biochemists}

Secr.: Mrs. P. Nielsen, Focus 98 Office, P.O. Box 227, GB-Buckingham MK18 5PN,

Tel.: (44) 1280860 613; Fax:(44) 1280860 487, E-Mail: 100673.33440@ compuserve.com,

Internet: http://www.Leeds.ac/UK/ACB

33rd Annual Meeting of the German Society of Diabetes

33. Jahrestagung der Deutschen Diabetes-Gesellschaft

Secr.: Ms. M. Twilfer, Hansisches Verlagskontor Lübeck, H. Scheffler, Mengstr. 16,

23552 Lübeck, Germany, Tel.: (49) 451703101 204; Fax: (49) 4517031253281

Spring National Meeting of the Professional Association of Physicians for

Microbiology and Infections Epidemiology

Frühjahrstagung des Berufsverbandes der Ärzte für Mikrobiologie \&

Infektionsepidemiologie

Secr.: Dr. med. W. Hauck, Nowakanlage 11, D-76137 Karlsruhe, Germany,

Tel.: (49) 721937 4690; Fax: (49) 7219374391

\section{5th World Congress on Biosensors}

Secr.: Phillipa Orme; Biosensors 98, The Boulevard, Langford Lane, Kidlington Oxford OW5 1GB, UK, Tel.: (44) 1865843 691; Fax: (44) 1865843958

\section{6th Nordic Congress of Clinical Chemistry - Laboratory Medicine 98}

Secr.: Congress Office / Laboratory Medicine 98, University of Turku, Lemminkäisenkatu 14 - 18 B, FIN-20520 Turku, Finland, Tel.: (358) 221333 6342; Fax: (358) 221333 6410, E-Mail: escon@utu.fi

\section{3th Congress of the German Society of Oncology}

23. Kongress der Deutschen Krebsgesellschaft

Secr.: Congress Project Management, Letzter Hasenpfad 61, D.60598 Frankfurt/Main, Germany, Tel.: (49) 696090 9531; Fax: (49) 6960909540

41st Hamburger Symposium on Coagulation 41. Hamburger Symposium über Blutgerinnung

Secr.: Prof. Dr. R. Matthias, Zentrum Innere Medizin, Klinikum der Justus Liebig-Universität, Klinikstrasse 36, D-35385 Gießen, Germany, Tel.: (49) 6419942 131; Fax: (49) 6419942139

15th International Conference on Human Tumor Marker Oncology (IATMO Meeting)

Secr.: Dr. Schlegel, Pharmamarketing AG, Sennweidstrasse 46, CH-6312 Steinhausen, Switzerland,

Tel.: (41) 71748 7600; Fax: (41) 71748 7611, E-Mail: h.fuchs@schlegelpharma.ch 


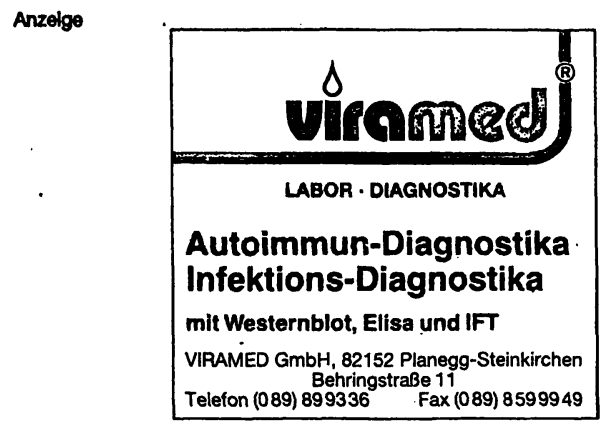

June 18 - 20, 1998

Wuppertal, Germany

June 19 - 20, 1998

Erfurt, Germany

June 20 - 22, 1998

Ljubljana, Slovenia

June 22 - 26, 1998

Ljubljana, Slovenia

June 28 - July 1,1998

Erfurt, Germany

June 28-July 3, 1998

Singapore, Singapore

June 28-July 3, 1998

Oslo, Norway

June 28-July 3, 1998

Geneva, Switzerland
41. Jahrestagung der Arbeitsgemeinschaft der Ärzte Staatlicher und Kommunaler Blutspendedienste

Secr.: Comed GmbH, Kongreßorganisation, Goethestrasse 8, D-791000 Freiburg, Germany,

Tel.: (49) 761 77740; Fax: (49) 761 74454, E-Mail: comed@t-online.de

7th Symposium on Atherosclerosis

7. Atherosklerose-Symposium

Secr.: Prof Dr. U. Till, Zentr. für Vasculäre Biologie und Medizin d. F. S.-Universität,

Nordhäuser Straße 78, D-99098 Erfurt, Germany, Tel.: (49) 3617411 203; Fax: (49) 3617411103

E-Mail: athsymp@zmkh.ef.uni-jena.de

44th Annual Meeting of the Scientific \& Standardization Committee (SSC) of the International Society on Thrombosis and Hemostasis - ISTH

Inform: Secretariat of the XIV ISFT: Cankarjev Dom, Congress and Cultural Centre, Presernova 10, SLO-1000 Ljubljana, Slovenia, Tel.: (386) 61 i1767 132; Fax: (386) 61217431 ,

E-Mail: Teja.Alic@cd-cc.si

14th International Congress on Fibrinolysis and Thrombolysis (ISFT)

Inform: Secretariat of the XIV ISFT: Cankarjev Dom - Congress and Cultural Centre. Presernova 10, SLO-1000 Ljubljana, Slovenia, Tel.: (386) 611767 135; Fax: (386) 61217431 ,

E-Mail: Teja.Alic@cd-cc.si

\section{7th National Meeting of Erfurt Conference of Platelets}

Secr.: Dr. W. Lösche, Centre for Vascular Biology and Medicine, Nordhäuser Straße 78, D-99089 Erfurt, Germany, Tel.: (49) 3617411 442; Fax: (49) 3617411 202, E-Mail: lvesche@zmkh.ef.uni-jena.de, Internet: www.uni-jena.de/epg

\section{3rd World Congress of Medical Technology}

Secr.: 23rd World Congress of Medical Technology c/o Academy of Medicine, Singapore, 16 College Road 01-01, Singapore 169854, Tel.: (65) 2238 968; Fax: (65) 2255155

25th Congress of the International Society of Blood Transfusion

Secr: DER - Deutsches Reisebüro, Mr. Schrank, Emil-von-Behring-Strasse 6, D-60439 Frankfurt,Germany, Tel.: (49) 69 9588.3610; Fax: (49) 6995883608

\section{International AIDS Conference}

Secr:Ms. D. Andrew, Office du Tourisme, P.O.Box 596, 10 route de l'Aeroport. Ch-1215 Geneva, Switzerland, Tel..(41) 22788 0808; Fax: (41) 227888170 
July 5 - 11, 1998

Baden-Baden, Germany

August 2 - 6, 1998

Chicago, IL. USA

August 22 - 26, 1998

Vienna. Austria

Aug. 30 - Sept. 4, 1998

Umea, Sweden

September 6 - 9, 1998

Geneva, Switzerland

September 8 - 12, 1998

Nürnberg, Germany

September 9 - 12, 1998

Dresden, Germany

September 14 - 16, 1998

Bremen, Germany

September 17 - 19, 1998

Regensburg, Germany

September 19 - 24, 1998

Erlangen, Germany

September 23 - 26, 1998

Freiburg, Germany

\section{5th MEDICONGRESS Baden-Baden 1998}

25. Seminarkongress für ärztliche Fortbildung

Secr: MEDICA Deutsche Gesellschaft zur Förderung der Medizinischen Diagnostik e. V.. P.O. Box 7001 49, D.70571 Stuttgart. Germany. Tel.: (49) 711763 443; Fax: (49) 711766992

50th National Meeting of the American Association of Clinical Chemistry (AACC) Americinn Association for Clinical Chemistry. Meetings Department, 2101 L Street. NW, Suite 202. Washington, DC 20037 - 1576, USA, 'Tel.: (1) 2028570717 ; Fax: (1) 2028335093 ,

E-Mail: custserv@aacc.org

\section{0th Congress of the European Society of Cardiology}

Secr.: The European Heart House (ECOR), 2035 Route des Colles - Les Templiers, P.O. Box 179, F-06903 Sophia Antipolis Cedex, France, Tel.: (33) 49294 7600; Fax: (33) 49294 7601, E-Mail: wcbmaster@escardio.org. Internet: http://www.escardio.org

26th Meeting of the International Society for Oncodevelopmental Biology \& Medicine (ISOBM)

Secr.: Prof. S. Hammerström, Dept. Immunology, Umea Universitiy, S-90185 Umea, Sweden, Tel.: (46) 907852345

70th Congress of the European Atherosclerosis Society (EAS)

Secr.: Prof. Y. Stein, P.O. Box 12249, Jerusalem 91120, Israel, Tel.: (972) 2677 6928; Fax: (972) 2643 4701, E-Mail: eranl@hadassah.org.il

52nd National Congress of the German Society of Gynaecology and Obstetrics 52. Kongreß der Deutschen Gesellschaft für Gynäkologie \& Geburtshilfe

Secr.: Prof. Dr. med. D. Berg, Congress Projekt Management GmbH , Letzter Hasenpfad 61, D-60598 Frankfurt/Main, Tel.: (49) 69609095 31; Fax: (49) 6960909540

32nd Annual Meeting of the German Society of Biomedical Techniques 32. Jahrestagung der Deutschen Gesellschaft für Biomedizinische Technik Secr.: Mrs. E. Toepfer, Techn. University Dresden, Institute of Biomedicine Technic, Momsenstr. 13, 01062 Dresden, Germany, Tel.: (49) 351463 5121; Fax: (49) 463 6026, E-Mail: etoepfer@rcs.urz.tu-dresden.de

43nd Annual Meeting of the German Society of Medical Informatics and Biometry 43. Jahrestagung der Deutschen Gesellschaft für Medizinische Informatik, Biometrie und Epidemiologie

Secr.: Dipl. Th. Banasiewicz, c/o BIPS, P.O. Box 106 767, D-28067 Bremen, Germany, Tel.: (49) 421219073 ; Fax: (49) 421216419

IFCC/Beckman European Conferences "Frontiers in Molecular Basis of Diseases" (1) Inflammatory Diseases

Secr.: Prof. Dr. Gerd Schmitz, University of Regensburg, D-93042 Regensburg, Germany,

Tel.: (49) 941 944620; Fax: (49) 941 9446202, E-Mail: gerd.schmitz@klinik.uni-regensburg.de

29th Congress of the German Society of Nephrology

31st Annual Meeting of the Working Group for Clinical Nephrology

29. Kongreß der Gesellschaft für Nephrologie

31. Jahrestagung der Deutschen Arbeitsgemeinschaft für klinische Nephrologie

Secr.: M. Schmidt, Medizinische Klinik IV, Friedr.-Alexander-Univ., Krankenhausstr.12,

D-91054 Nürnberg, Germany, Tel.: (49) 911859 002; Fax: (49) 911859 209,

E-Mail: mon@mcn-nuernberg.de

29th Conference of the German Society of Immunology

29. Tagung der Deutschen Gesellschaft für Immunologie

Secr.: Prof. Dr. H. Peter, Klinikum der Albrecht-Ludwig-Universität, Rheumatologie,

Hugstetter Str. 55, D-79106 Freiburg, Tel.: (49) 761270 3448; Fax: (49) 7612703446 
September 24 - 26, 1998

Karlovy Vary (Carlsbad), Czech Republic

Sept. 30 - Oct. 4, 1998

Estoril, Portugal

October 5 - 8, 1998

Berlin, Germany

October 6 - 9, 1998

Bern, Switzerland

October 8 - 10, 1998

Berlin, Germany

October 25 - 28, 1998

Frankfurt, Germany

November 15 - 18, 1998

Düsseldorf, Germany.

November 18 - 21, 1998

Düsseldorf, Germany

\section{5th Alpe-Adria Congress of Clinical Chemistry}

Secr.: Prof. Dr. P. Schneiderka, Dept. of Clinical Biochemistry, 1st Medical Faculty, Charles University, Karlovo n. 32, CZ-121 11 Praha 2, Czech Republic. Tel./ Fax: (420) 2291770,

E-Mail: pschne@if1.cuni.cz

7th International Congress on Pediatric Laboratory Medicine

Secr.: Luxor - Dr. Judite Delgado. Av. da Republica, $\mathrm{n}^{\circ} 62-2^{\circ} \mathrm{DT}^{\circ} ., 1050$ Lisboa, Portugal, Tel.: (351) 1795 1546; Fax: (351) 1796 2084, E-Mail: luxor@mail.telepac.pt

50th National Congress of the German Society of Hygiene \& Microbiology 50. Kongreß der DGHM - Deutsche Gesellschaft für Hygiene \& Microbiologie

Secr.: Prof. Dr. Dr. U. Göbel, Institut für Microbiologie und Hygiene, Universitätsklinikum Charité, Dorotheenstraße 96, D-10117 Berlin, Germany, Tel.: (49) 302093 4715; Fax: (49) 302292741

31st Annual Congress of the German Society of Transfusion Medicine \& Immunohematology

31. Jahreskongress der Deutschen Gesellschaft für Transfusionsmedizin \& Immunhämatologie

Secr.: Prof. Dr. U. Nydegger, Hämatologiesches Zentrallabor, Inselspital, CH-3010 Bern. Switzerland, Tel.: (41) 316322 111; (41) 31 6359 366, Congress-Office Lentzsch GmbH, E-Mail: lentzsch@ad.com

Update in Thrombolysis 1998

Annual Meeting on Thrombolysis, Arteriosclerosis, Thrombosis \& Cardiovascular Biology

Secr.: Prof. D. C. Gulba, Virchow-Hospitals, Humboldt-University, Franz-Vollhard-Hospital, Wiltbergstraße 50, D-13125 Berlin, Germany, Tel.: (49) 309417 2400; Fax: (49) 3094172453 , E-Mail: porstmann@t-online.de

Joint Annual Meeting of the German \& Austrian Society of Haematology \& Oncology

Gemeinsame Jahrestagung der Deutschen \& Österreichischen Gesellschaft für Hämatologie \& Onkologie

Secr.: K.I.T. GmbH, Kongreß- und Incentive Organisation, Wilhelm-Leuschner-Straße 43 , D-60239 Frankfurt/Main, Germany, Tel.: (49) 692427 9067; Fax: (49) 6924279068

Joint Congress of the German Society of Laboratory Medicine and the German Society of Clinical Chemistry - 1998 .

Kongreß für Laboratoriumsmedizin 1998

Info.: Deutsche Gesellschaft zur Förderung der Medizinischen Diagnostik e.V., MEDICA e.V., P.O. Box 7001 49, D-70571 Stuttgart, Germany, Tel.: (49) 711 765 1454; Fax: (49) 711766992

MEDICA '98: The 30th Annual International Meeting MEDICA: Weltforum für Arztpraxis und Krankenhaus 30. Internationale Fachmesse und Kongreß

- Info.: Deutsche Gesellschaft zur Förderung der Medizinischen Diagnostik e.V., MEDICA e.V., P.O. Box 7001 49, D-70571 Stuttgart, Germany, Tel.: (49) 711765 1454; Fax: (49) 711766992
February 1 - 3, 1999

Tutzing, Germany.

February 3 - 5, 1999

Paris, France
IFCC Bergmeyer Conference

Biochemical Markers for Myocardial Damage: Current Status, Future Trends

Secr.: Ms. Chantal Thirion, IFCC Secretariat, Centre du Médicament, Université de Nancy, 30. Rue Lionnois. F-54000. Nancy, France, Tel.: (33) 38335 2616; Fax: (33) 383321322

4th International Fibrinogen Symposium: Fibrinogen \& Cardiovascular Diseases Secr.: c/o Prof. L. O. Drouet, Institut des Vaisseaux et du Sang, Hôpital Lariboisière, 2 rue Ámbroise Paré, F-75475 Paris, France, Tel.: (33) 1452621 98; Fax: (33) 142829473 
February 24 - 27. 1999 Mannheim. Germany

June 6 - 11, 1999

Florence, Italy

July 25 - 29, 1999

New Orleans, LA, USA

August 14 - 21, 1999

Washington, DC, USA

September 4 - 8, 1999

Barcelona, Spain

October 17 - 21, 1999

Lahore, Pakistan

November 8 - 11, 1999

Atlanta, GA, USA

December 3 - 7, 1999

New Orleans, LA, USA 43rd Annual Meeting of the Society on Thrombosis \& Haemostasis Research 43. Jahrestagung der Gesellschaft für Thrombose- und Hämostaseforschung

Secr.: Prof.: Dr. D. L. Heenc, Universitätsklinikum Mannheim. Ruprecht-Karls-Universität Heidelberg, Theodor-Kutzer-Ufer 1-3, D-68167 Mannhcim, Tel.: (49) 621383 2202; Fax: (49) 6213832153.

E-Mail: dictcrhecne@medl.ma.uni-heidelherg.de

\section{7th International \& 13th European Congress of Clinical Chemistry /} IFCC-World Lab 99

Organizing Secretariat XVII ICCC '99. Emmezeta S.r.l., Via C. Farini, 70, I-20159 Milan, Italy, Tel.: (39) 26680 2323; Fax: (39) 2668 6699, E-Mail: emmezeta@galactica.it

51st National Meeting of the American Association of Clinical Chemistry (AACC) American Association for Clinical Chemistry, Meetings Department, 2101 L Street, NW, Suite 202. Washington, DC 20037 - 1526, USA, Tel.: (1) 202857 0717; Fax: (1) 2028334576

17th Congress of the International Society on Thrombosis and Haemostasis (ISTH) 45th Annual Meeting of the Scientific \& Standardization Committee

Secr.: Prof. Victor J. Marder, University of Rochester Medical Center, Hematology Unit, Box 610,601 Elmwood Avenue, Rochester, New York 14642, USA,

Tel.: (1) 716275 3761; Fax: (1) 716473 4314; E-Mail: victor_marder@medicine.rochester.edv

21st Congress of the European Society of Cardiology

Secr.: The European Heart House (ECOR), 2035 Route des Colles - Les Templiers, P.O. Box 179, F-06903 Sophia Antipolis Cedex, France, Tel.: (33) 49294 7600; Fax: (33) 492947601

\section{2th Asian Pacific Congress of Cardiology}

Secr.: Pakistan Cardiac Society. XIIth APCC, Punjab Institute of Cardiology. Ghaus-ul-Azam Road. Lahore, Pakistan-54000, Tel.: (92) 42758 0827; Fax: (92) 42758 5899; E-Mail: pic@aster.com.pk; Internet: http://www.aster.com.pk./pic.htm

\section{American Heart Association - 72nd Scientific Sessions}

American Heart Association, Scientific and Corporate Meetings, 7272 Greenville Avenue, Dallas, TX 75231-4596, USA, Tel.: (1) 214706 1511; Fax: (1) 214373 3406; Internet: http://www.amhrtorg

41st Annual Meeting of the American Society of Hematology

Dr. Donald Feinstein, University of Southern California, School of Medicine, 2025 Zonal Avenue, Los Angeles, CA 90033, USA, Tel.: (1) 213224 7224; Fax: (1) 2132251258 